\title{
The upgraded ISOLDE yield database - A new tool to predict beam intensities
}

Ballof, J.

2020-01-15

Ballof , J , Ramos , J P , Molander , A , Johnston , K, Rothe , S , Stora , T \& Duellmann , C E 2020 , ' The upgraded ISOLDE yield database - A new tool to predict beam intensities ' , Nuclear Instruments \& Methods in Physics Research. Section B: Beam Interactions with Materials and Atoms , vol. 463 , pp. 211-215 . https://doi.org/10.1016/j.nimb.2019.05.044

http://hdl.handle.net/10138/338774

https://doi.org/10.1016/j.nimb.2019.05.044

cc_by_nc_nd

acceptedVersion

Downloaded from Helda, University of Helsinki institutional repository.

This is an electronic reprint of the original article.

This reprint may differ from the original in pagination and typographic detail.

Please cite the original version. 


\title{
The upgraded ISOLDE yield database - A new tool to predict beam intensities
}

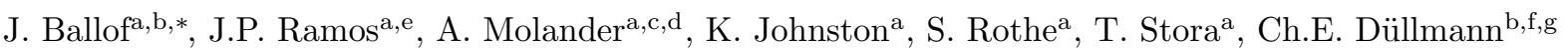 \\ ${ }^{a}$ CERN, 1211 Geneva 23, Switzerland \\ ${ }^{b}$ Johannes Gutenberg - Universität Mainz, Institut für Kernchemie, Fritz-Strassmann-Weg 2, 55128 Mainz, Germany \\ ${ }^{c}$ University of Helsinki, FI-00014 Helsinki, Finland \\ ${ }^{d}$ Helsinki Institute of Physics, P. O. Box 64, FI-00014 Helsinki, Finland \\ ${ }^{e} K U$ Leuven, Instituut voor Kern- en Stralingsfysica, B-3001 Leuven, Belgium \\ ${ }^{f}$ GSI Helmholtzzentrum für Schwerionenforschung, 64291, Darmstadt, Germany \\ ${ }^{g}$ Helmholtz-Institut Mainz, 55099 Mainz, Germany
}

\begin{abstract}
At the CERN-ISOLDE facility a variety of radioactive ion beams are available to users of the facility. The number of extractable isotopes estimated from yield database data exceeds 1000 and is still increasing. Due to high demand and scarcity of available beam time, precise experiment planning is required. The yield database stores information about radioactive beam yields and the combination of target material and ion source needed to extract a certain beam along with their respective operating conditions. It allows to investigate the feasibility of an experiment and the estimation of required beamtime. With the increasing demand for ever more exotic beams, needs arise to extend the functionality of the database and website not only to provide information about yields determined experimentally, but also to predict yields of isotopes, which can only be measured with sophisticated setups. For the prediction of yields, in-target production and information about release properties of target materials must be known. While the former were estimated in a simulation campaign using FLUKA and ABRABLA codes, the latter is available from measurement data as already stored in the database. We have compiled the information necessary to predict yields, and made available a yield prediction tool as web application. This currently undergoes extensive testing and will be available as powerful tool to the ISOLDE user community.
\end{abstract}

Keywords: CERN, ISOLDE, Radioactive Beams, Database, Yields, Yield Prediction, Cross sections, FLUKA, ABRABLA, Release efficiency, Production Yield, In-target production

PACS: 29.25.Ni, 29.25.Rm, 29.27.a, 29.50.+v, 13.85.Lg, 07.05.Tp

\section{Introduction}

The ISOLDE facility is one of the oldest experiments at CERN and has been continuously upgraded over the years [1. Today, it offers online production, extraction and sep5 aration of more than 1000 radioisotopes of 74 different chemical elements from thick targets, which are typically ${ }_{25}$ made of pressed powders, metallic foils, or molten materials. Within the target and ion source unit, the target materials are enclosed in a tantalum container, and are 10 coupled to a transfer line and ion source, which are also selected out of a variety of different types of sources and lines.

In earlier days, a comprehensive collection of yield data was available in the ISOLDE Users' guide compiled by H.-

15 J. Kluge [2]. Later, an online yield database was developed to provide information about yields to users and technical teams [3, 4, 5. It is now fully redesigned, upgraded using state-of-the-art technologies, extended in its functionality

\footnotetext{
* Corresponding author

Email address: Jochen.Ballof@cern.ch (J. Ballof)
}

and new yield data were included. The database and web 20 application [6] not only store experimental yield information, but also provide release properties, in-target production cross sections estimated by means of time consuming and computationally expensive Monte-Carlo simulations and algorithms to predict yields of isotopes which have not yet been measured.

\section{Beam Production at ISOLDE}

The isotope extraction and beam production from thick targets is a multistage process. The radioisotopes are produced upon impact of the primary $1.4-\mathrm{GeV}$ proton beam on a target material. The atoms then need to diffuse out of the target material and into the ion source, where they are ionized, extracted electrostatically and transported ion optically to an electromagnetic dipole for separation by mass to charge ratio..

An efficiency is associated to each step of the process. The first factor contributing to the radioactive ion beam yield $Y$ is the in-target production $N_{0}$. Efficiencies for beam transport to the focal plane of the magnet, ioniza- 


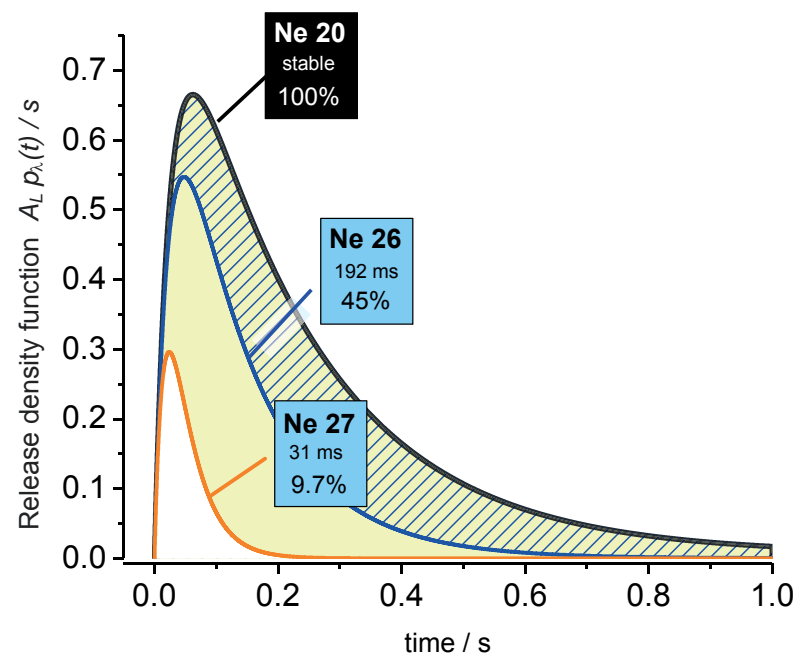

Figure 1: Typical non-normalized release curves $A_{L} p(t)$ of stable neon, and release curves $A_{L} p_{\lambda}(t)=A_{L} p(t) \mathrm{e}^{-\lambda t}$ of radioactive isotopes. The release efficiencies are given in percent and have been calculated according to Eq. 1] Parameters $t_{\text {rise }}, t_{\text {fall1 }}, t_{\text {fall2 }}$ and $\alpha$ (cf. Eq. 2] taken from Ref. [10]. The normalization parameter $A_{L}$ has to be chosen such, that $\int_{0}^{\infty} p(t) \mathrm{d} t$ computes to unity.

tion and chemical efficiency can be summarized in an over-

all efficiency parameter $\epsilon_{\mathrm{f}}$. The half-life dependent release efficiency $\left(\epsilon_{\text {release }}\right)$ is governed by diffusion and effusion processes and takes into account the time passing between production and release. A more detailed discussion of efficiency parameters can be found e.g. in Ref. [7].

Each atom follows its individual path to reach the ion 75 source, and the distribution function for the time needed to follow these trajectories is defined by the release density function $p(t)$ [8, 9, The release efficiency of an isotope with decay constant $\lambda$ is obtained by folding the release density function $p(t)$ with a factor to account for decay losses.

$$
\begin{aligned}
& Y=N_{0} \epsilon_{\text {release }} \epsilon_{\mathrm{f}}, \text { where } \\
& \epsilon_{\text {release }}=\int_{0}^{\infty} p(t) e^{-\lambda t} \mathrm{~d} t
\end{aligned}
$$

The release density function is normalized such, that ${ }_{80}$ $\int_{0}^{\infty} p(t) \mathrm{d} t=1$. The efficiency parameter $\epsilon_{\mathrm{f}}$ and the distribution function $p(t)$, which describes the release of a stable nuclide, depend on the chemical element, operating conditions and typically only to negligible extend on the isotope. Hence, it is only required to experimentally ${ }_{85}$ determine the parameters $\epsilon_{\mathrm{f}}$ and $p(t)$ once for each chemical element in a defined target and ion source system at given operating conditions. Knowing the two parameters 60 and the in-target production rates of isotopes, allows to predict yields for the full isotopic chain (cf. Eq. 11). The 90 isotope for the measurement of $p(t)$ must be chosen such, that the tail of the experimentally obtained radioactive release curve $p_{\lambda}(t)=p(t) \mathrm{e}^{-\lambda t}$ is not governed by radioactive 65 decay.

From elementary diffusion and effusion processes, ex- 95 pressions for the delay function $p(t)$ have been obtained,

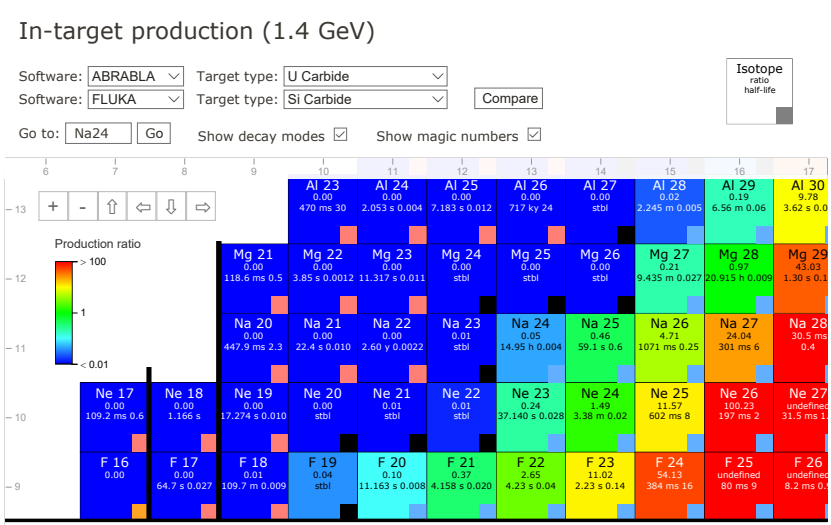

Figure 2: Cut-out of the interactive nuclide chart, which is used in the web application to visualize in-target production and radioactive beam yields. The example is meant to demonstrate the capabilities of the software and shows the relative in-target production rates of two different target materials estimated by simulations using two different codes. The background color represents the production ratio, and the inset rectangle the decay mode of the isotope. In addition, half-life information is given.

e.g. by Kirchner et al. [8]. However, these expressions depend on numerous parameters, such as target material properties, target geometry and operation conditions. In addition, the expressions are often difficult to fit to experimental data. For practical purposes, the experimental data is often fitted to the three exponential function given in Eq. 2 [12, where $A_{L}$ is a normalization parameter, $t_{\text {rise }}, t_{\text {fall1 }}$ and $t_{\text {fall2 }}$ are time constants describing the curve shape, and $\alpha$ is a weighting coefficient.

$$
\begin{aligned}
p(t)= & \frac{1}{A_{L}}\left(1-\mathrm{e}^{-t \ln (2) / t_{\text {rise }}}\right) \\
& \cdot\left(\alpha \mathrm{e}^{-t \ln (2) / t_{\text {fall1 } 1}}+(1-\alpha) \mathrm{e}^{-t \ln (2) / t_{\text {fall } 2}}\right)
\end{aligned}
$$

A typical release curve of stable neon is shown in Fig. 1. along with calculated release efficiencies for short-lived radioisotopes. At the time of writing, release curves for 427 yield entries are available in the yield database.

A common procedure to assess a yield, is based on the measurement of a release curve, as shown in Fig. 1 . ISOLDE is supplied with protons from the proton synchrotron booster (PSB), which arrive as narrow pulses of only a few microseconds length and typically contain up to $3 \times 10^{13}$ protons. The proton pulse impinges on the target at $t=0$. After a certain delay time $t=t_{\text {delay }}$, electrostatic deflectors allow the radioactive beam emitted from the ion source to pass downstream the beamline and to the tape of the tape station, in which it is implanted for a defined collection time $t_{\text {collect }}$. After the end of the collection $\left(t=t_{\text {delay }}+t_{\text {collect }}\right)$, the radioactive ion beam transport is prevented by electrostatic deflectors, and the tape is forwarded to move the collected activity to the measurement position, where it is counted by calibrated detectors. Taking into account the decay during tape transport, the num- 
ber of ions per second extracted at $t=t_{\text {delay }}+0.5 t_{\text {collect }}$ is obtained. Repeating the procedure whilst varying the ${ }_{150}$ delay time $t_{\text {delay }}$ allows to sample a full release curve of the radioactive beam. The yield in the focal center of the separation magnet is obtained by integration of the nonnormalized release curve, and correcting for losses by beam transport from magnet to tape station. Beam losses are estimated by measurement of stable beam with Faraday Cups placed along the beamlines. The procedure is summarized in Ref. [3, and more detail about pulse shapes, yield calculation and efficiencies is given in Lit. [27.

\section{In-Target Production}

The in-target production rates $N_{0}$ of all common target materials were assessed by means of an extensive simulation campaign. High statistics of up to $1 \times 10^{9}$ primary particles are needed to achieve reliable production rates also165 for exotic nuclides. Two simulation codes have been used to investigate the production rates. The ABRABLA code 13 is commonly used at ISOLDE and was already used and benchmarked for $600 \mathrm{MeV}$ and $1.0 \mathrm{GeV}$ proton beam 14. Per each simulation run, the code yields radionu-170 clide production cross sections at a defined driver beam energy and target nuclide, and is therefore ideally suited the target, where secondary reactions do not contribute significantly. The cross section estimates are obtained at $t_{175}$ the initial energy of the proton beam, not taking into account energy losses in the target. The particle transport FLUKA 15, 16 allows the definition of complex geometries and calculates multiple particle interactions with matter using various integrated physics models. The def-180 inition of a geometry has especially proven useful for target units equipped with a neutron spallation source, the so called proton-to-neutron converter [17, 18].

The codes were used to simulate isotope production with common and prospective target materials at proton ${ }_{185}$ beam energies of $0.6,1.0,1.4$ and $2.0 \mathrm{GeV}$, to take into account historic driver beam energies delivered by the Syn-

135 chrocyclotron (SC) along with the early, present and future beam energies of the Proton Synchrotron Booster (PSB), which supplies ISOLDE [19. Besides the prediction of exotic nuclide yields, the simulation data allows the estimation of release efficiencies if experimental yields of several isotopes of the same chemical element are available. Thus, it gives insight into release properties of targets and ion sources used for the past 60 years at ISOLDE. The data enabled us to conduct a systematic study of isotope pro- ${ }_{195}$ duction at the planned upgraded driver beam energy of

\section{Technical Implementation}

All software components have been developed compat- 200 ible to the CERN centrally provided IT infrastructure ser-

vices to ensure reliability and long-term support. The Oracle Database 11g 20, which is widely used at CERN, serves as underlying data provider for all high-level applications.

\subsection{Database}

The database stores experimentally obtained radioactive ion beam yields which were typically measured using the ISOLDE tapestation and taken from publications. The yields are stored along with information about the used target-unit, such as type of ion source, target material, thickness and operational conditions, and are given as yield in the focal plane of the magnet. Within this work, the structure of the database was completely redesigned and further normalized to ease maintenance and allow data storage for the upgraded functionality. A measured yield is linked to a defined target unit, which in turn is associated with a target material, made up of a nuclide mixture in defined stoichiometry. A set of new tables was added to store in-target production data obtained in sumulations. For each target material nuclide, one ABRABLA run is necessary. The total in-target production cross section of a target material is then calculated by combining the estimated cross sections obtained in multiple ABRABLA runs and the thickness (areal density) of the target unit. In addition, FLUKA simulation results are available. Here the full target geometry and nuclide inventory is covered by one simulation. Release curve parameters are required to calculate release efficiencies (cf. Eq. 2) and are available in a table linked to the table holding information about measured yields. At the time of writing, ca. one million production cross sections estimated by simulation codes are available, also indicating the production channel (like fission or spallation) for ABRABLA data. Besides storing published yield information, the database was extended to maintain data of yield measurements, typically taking place before each physics run. Within the 2018 operating period, new yields have been introduced, which are now available to advanced users.

\subsection{User and Application Programming Interfaces}

Two new user-interfaces have been developed. One interface is implemented as website and displays data to users. For fast data manipulation, a rich client solution is under development. A prototype application implemented using Microsoft Access is already provided to users as RemoteApp by Microsoft Remote Desktop services (RDS) 21] to allow a rich client experience but avoiding at the same time the need of local software installation on client computers.

\subsubsection{Web Application}

In contrast to earlier versions, the new web application [6] is not based on Oracle integrated web solutions and Java applets, but implemented in C\# under Microsoft 


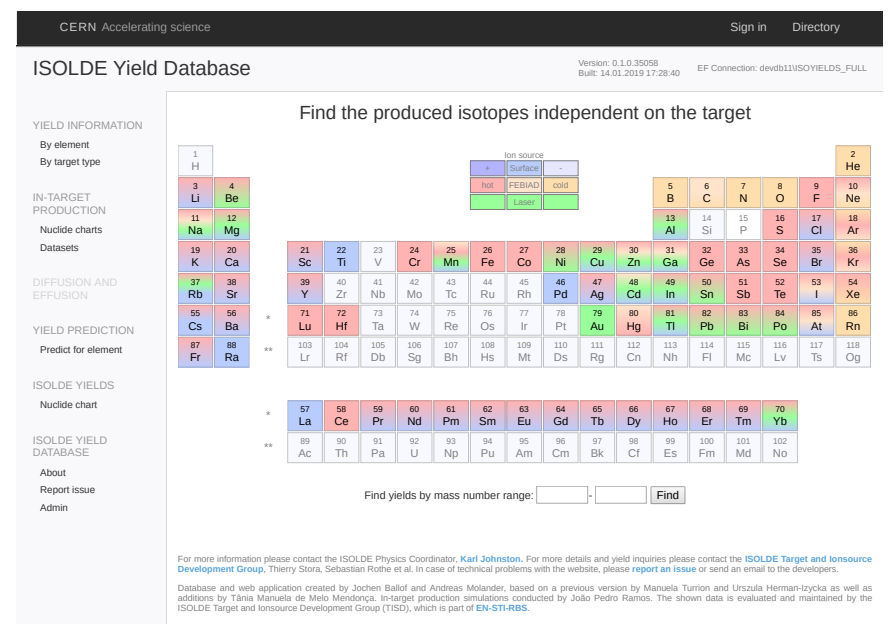

Figure 3: The web frontend of the ISOLDE yield database, showing available beams in the periodic table of elements. The frontend is available at http://cern.ch/isolde-yields.

ASP.NET 22] to gain a higher degree of flexibility in development. User authentication is accomplished by Single Sign On (SSO) services, widely used at CERN. The Microsoft Entity Framework [23] allows object-relational mapping and rapid development of data driven applica-24 tions. With the new application, further details about production conditions of yields are given, which include among other attributes, target and ion-source temperatures and release curves. The latter are provided as parameters (cf. Eq. 2) and plot to visualize the time structure of the release. Besides adding yield details, an interactive 245 nuclide chart was developed using the JavaScript library D3.js [24], which is capable of displaying an overview chart of all beams at ISOLDE as well as in-target production yields for each target material [25]. For easy comparison between target materials or different driver beam energies, 250 the plotting of ratios between different data sets is also available (cf. Fig. 2). The functionality to predict yields for not yet measured isotopes is also included in the web application. It allows the user to predict yields for the full chain of isotopes of a certain element. The desired target material, proton beam energy and simulation software are selected by the users. The application then searches ${ }_{255}$ for reference point candidates in the database, which serve as source for the calculation of the efficiency parameter $\epsilon_{\mathrm{f}}$. The actual reference point is selected by the user, and prediction results are displayed as table and plot. A typical plot is shown in Fig. 4. Half-life data of nuclides is nec- ${ }_{260}$ essary to calculate release efficiency. An import function for NUBASE evaluation data [26] allows to continuously update the data with new and modified entries.

\subsubsection{Rich Client Application}

The prototype desktop application allows fast and direct access to the data layer. It is intended to be used for data manipulation and queries by advanced users. Functionality has been included to calculate yields obtained

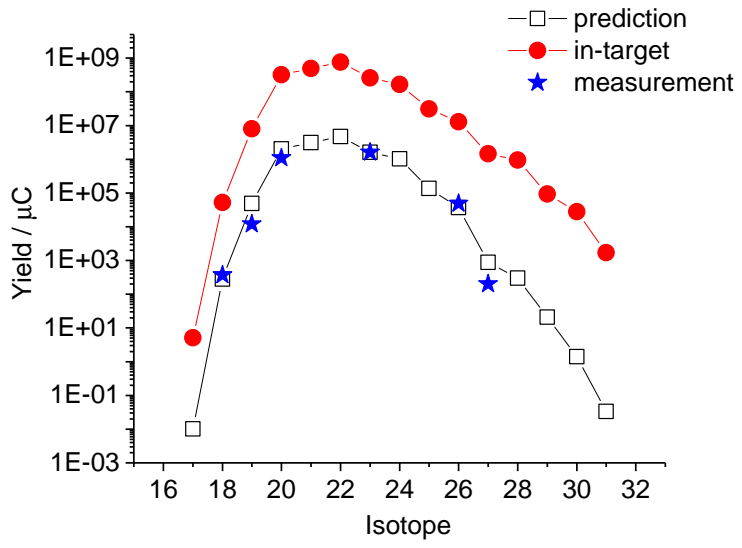

Figure 4: Exemplary plot showing measured yields, in-target production along with yield predictions of neon isotopes from a uranium carbide target [10, 6]. The experimental yield for ${ }^{23} \mathrm{Ne}$ was used as reference point to calculate $\epsilon_{\mathrm{f}}$. The in-target production yields were taken from ABRABLA.

during routinely performed measurements for each unit, which are either conducted at the ISOLDE tape station or derived from Faraday Cup readings. The tape station is equipped with scintillation counter and high purity germanium detector and used as reference method to determine yields.

\subsubsection{Application Programming Interface}

Following user requests to link their own systems to the yield database, a REST-interface based on the ASP.NET Web API is available. The solution integrates nicely into Entity Framework and enables us to rapidly develop programming interfaces. The data is delivered as JSON or XML data. The interface is also used to provide data to the CRIBE (Chart of Radioactive Ion Beams in Europe) project, which aims at providing an overview of available and future radioactive ion beams at all facilities in Europe [28.

\section{Conclusions and Outlook}

Within a complete redevelopment of the website and restructuring of the underlying database we have added a set of new functions to the ISOLDE yield database application. Now, it does not only provide available yield information, but is also capable to predict yields at the prospective proton beam energy of $2.0 \mathrm{GeV}$, which is an option of the driver beam upgrade under consideration. Yield predictions for exotic isotopes, which could only be measured with sophisticated setups, are now available. To achieve the latter, a comprehensive simulation campaign was conducted. The results of the simulation data are made available to users. At the time of writing the application is available to advanced users and undergoes extensive testing before release on the ISOLDE web page. In the future, we plan to further extend the functionality 
270 of the database and develop interfaces for data exchange between asset management and control applications. The ${ }^{330}$ inclusion of yields reported by facility users and an extension of the simulation campaign are in preparation. The added simulation data will cover cross sections of materi-

275 als which have not yet been used at ISOLDE, and will also ${ }^{335}$ be verified against new versions of the simulation codes.

\section{Acknowledgments}

We would like to thank T.M. Mendonça for her comprehensive revision of available yield information, evaluation of user requirements, and work towards the redesign of the webpage, R. S. Augusto for support regarding the FLUKA ${ }^{345}$ code, as well as A. Kelic-Heil and J. Klimo for ABRABLA support. We also thank M. Rey Regulez, I. Posada Trobo and the CERN IT staff for fast and reliable hosting of database and web application and acknowledge continuous support. Regarding the implementation of the CRIBE interface, we appreciate the fruitful collaboration with M. Celary and M. Fadil (GANIL). Moreover, we thank M. J. García Borge, G. Neyens and the members of the ISOLDE physics group for their support and valuable input. This project has received funding from the European Union's Horizon 2020 research and innovation program under grant ${ }_{360}$ agreement No 654002.

\section{References}

[1] R. Catherall, W. Andreazza, M. Breitenfeldt, A. Dorsival, G. J. Focker, T. P. Gharsa, G. T. J, J.-L. Grenard, F. Locci, P. Martins, S. Marzari, J. Schipper, A. Shornikov, T. Stora, The isolde facility, Journal of Physics G: Nuclear and Particle Physics 44 (9) (2017) 094002. URL http://stacks . iop.org/0954-3899/44/i=9/a=094002

[2] H. J. Kluge, ISOLDE users' guide, CERN Yellow Reports: Monographs, CERN, Geneva, 1986. URL http://cds.cern.ch/record/170359

[3] M. Turrión, M. Eller, R. Catherall, L. Fraile, U. Herman-375 Izycka, U. Köster, J. Lettry, K. Riisager, T. Stora, Management of isolde yields Nuclear Instruments and Methods in Physics Research Section B: Beam Interactions with Materials and Atoms 266 (19) (2008) 4674 - 4677, proceedings of the XVth International Conference on Electromagnetic Iso-380 tope Separators and Techniques Related to their Applications. doi:https://doi.org/10.1016/j.nimb.2008.05.144

I. URL http://www.sciencedirect.com/science/article/pii/ S0168583X08007994

[4] M. Turrión, M. Borge, O. Tengblad, Database for ISOL- 385 production optimization with web access Nuclear Physics A 746 (2004) 441-444. doi:10.1016/j.nuclphysa.2004.09.062 URL https://doi.org/10.1016/j.nuclphysa.2004.09.062

[5] The ISOLDE yield database - Classic version, http:// www.isoyields-classic.web.cern.ch [Online; accessed 14-390 December-2018] (2018).

[ [6] The ISOLDE yield database - Development version, http:// www.isoyields2.web.cern.ch [Online; accessed 14-December2018] (2018).

[7] U. Köster, How to produce intense and pure ISOL beams 395 Progress in Particle and Nuclear Physics 46 (1) (2001) 411 412. doi:https://doi.org/10.1016/S0146-6410(01)00148-X URL http://www.sciencedirect.com/science/article/pii/ S014664100100148X
[8] R. Kirchner, On the release and ionization efficiency of catcherion-source systems in isotope separation on-line, Nuclear Instruments and Methods in Physics Research Section B: Beam Interactions with Materials and Atoms 70 (1-4) (1992) 186-199.

[9] T. E. Cocolios, B. A. Marsh, V. N. Fedosseev, S. Franchoo, G. Huber, M. Huyse, A. M. Ionan, K. Johnston, U. Köster, Y. Kudryavtsev, M. Seliverstov, E. Noah, T. Stora, P. V. Duppen, Resonant laser ionization of polonium at rilis-isolde for the study of ground- and isomer-state properties Nuclear Instruments and Methods in Physics Research Section B: Beam Interactions with Materials and Atoms 266 (19-20) (2008) 44034406. doi:10.1016/j.nimb.2008.05.142

URL https : //doi . org/10.1016/j.nimb.2008.05.142

[10] U. Bergmann, G. Auböck, R. Catherall, J. Cederkäll, C. Diget, L. Fraile, S. Franchoo, H. Fynbo, H. Gausemel, U. Georg, T. Giles, H. Jeppesen, O. Jonsson, U. Køster, J. Lettry, T. Nilsson, K. Peräjärvi, H. Ravn, K. Riisager, L. Weissman, J. Aystö, Production yields of noble-gas isotopes from isolde ucx/graphite targets, Nuclear Instruments and Methods in Physics Research Section B: Beam Interactions with Materials and Atoms 204 (2003) 220 - 224, 14th International Conference on Electromagnetic Isotope Separators and Techniques Related to their Applications. doi:https://doi.org/10.1016/S0168-583X(02) 01921-3

URL http://www.sciencedirect.com/science/article/pii/ S0168583X02019213

[11] G. C. Everest, Basic data structure models explained with a common example, in: Proc. Fifth Texas Conference on Computing Systems, Computer Society Publications Austin, TX (Oct.). Long Beach, CA, 1976, pp. 18-19.

[12] J. Lettry, R. Catherall, P. Drumm, P. Van Duppen, A. Evensen, G. Focker, A. Jokinen, O. Jonsson, E. Kugler, H. Ravn, et al., Pulse shape of the isolde radioactive ion beams, Nuclear Instruments and Methods in Physics Research Section B: Beam Interactions with Materials and Atoms 126 (1-4) (1997) 130134.

[13] A. Kelic̀, M. V. Ricciardi, K.-H. Schmidt, Abla07 - towards a complete description of the decay channels of a nuclear system from spontaneous fission to multifragmentation, in: Joint ICTP-IAEA Advanced Workshop on Model Codes for Spallation Reactions Trieste, Italy, February 4-8, 2008, 2009. arXiv: 0906.4193

[14] S. Lukic̀, F. Gevaert, A. Kelic̀, M. Ricciardi, K.-H. Schmidt, O. Yordanov, Systematic comparison of isolde-sc yields with calculated in-target production rates, Nuclear Instruments and Methods in Physics Research Section A: Accelerators, Spectrometers, Detectors and Associated Equipment 565 (2) (2006) 784 - 800. doi:https://doi.org/10.1016/j.nima.2006.04. 082

URL http://www.sciencedirect.com/science/article/pii/ S0168900206007005

[15] A. Ferrari, P. R. Sala, A. Fass, J. Ranft, FLUKA: A multiparticle transport code (program version 2005), CERN Yellow Reports: Monographs, CERN, Geneva, 2005. URL http://cds.cern.ch/record/898301

[16] T. Böhlen, F. Cerutti, M. Chin, A. Fassó, A. Ferrari, P. Ortega, A. Mairani, P. Sala, G. Smirnov, V. Vlachoudis, The fluka code: Developments and challenges for high energy and medical applications Nuclear Data Sheets 120 (2014) $211-214$. doi:https://doi.org/10.1016/j.nds.2014.07.049.

URL http://www.sciencedirect.com/science/article/pii/ S0090375214005018

[17] R. Catherall, J. Lettry, S. Gilardoni, U. Köster, Radioactive ion beams produced by neutron-induced fission at isolde $\mathrm{Nu}-$ clear Instruments and Methods in Physics Research Section B: Beam Interactions with Materials and Atoms 204 (2003) 235 - 239, 14th International Conference on Electromagnetic Isotope Separators and Techniques Related to their Applications. doi:https://doi.org/10.1016/S0168-583X (02) 01915-8 URL http://www.sciencedirect.com/science/article/pii/ S0168583X02019158 
400 [18] T. Stora, E. Noah, R. Hodak, T. Y. Hirsh, M. Hass, V. Kumar, K. Singh, S. Vaintraub, P. Delahaye, H. Franberg-Delahaye, M.-G. Saint-Laurent, G. Lhersonneau, A high intensity 6he beam for the beta-beam neutrino oscillation facility EPL (Euro-

physics Letters) 98 (3) (2012) 32001. doi:10.1209/0295-5075/ $405 \quad 98 / 32001$

URL https://doi.org/10.1209/0295-5075/98/32001

[19] K. Hanke, Past and present operation of the CERN PS booster International Journal of Modern Physics A 28 (13) (2013) 1330019. doi:10.1142/s0217751×13300196

URL https://doi.org/10.1142/s0217751x13300196

[20] Oracle Corporation Website, http://www.oracle.com [Online; accessed 13-December-2018] (2018).

[21] Remote Desktop Services, https://docs.microsoft.com/

II en-us/windows-server/remote/remote-desktop-services/

415 welcome-to-rds/ [Online; accessed 15-December-2018] (2018).

[22] Microsoft ASP.NET documentation, https://docs.microsoft . com/en-us/aspnet/, [Online; accessed 14-December-2018] (2018).

[23] Microsoft Entity Framework documentation, https: //docs.microsoft.com/en-us/aspnet/, [Online; accessed 14-December-2018] (2018).

[24] M. Bostock, D3 - Data Driven Documents JavaScript library, https://d3js.org/ [Online; accessed 15-December-2018] (2010 -2017 ).

425 25] A. Molander, Development of the ISOLDE Yield Database, CERN Summer Student Programme Report URL http://cds . cern. ch/record/2636715

[26] G. Audi, F. G. Kondev, M. Wang, W. Huang, S. Naimi, The NUBASE2016 evaluation of nuclear properties, Chinese Physics C 41 (3) (2017) 030001. doi:10.1088/1674-1137/41/3/030001 URL https ://doi .org/10.1088/1674-1137/41/3/030001

[27] J. Lettry, R. Catherall, P. Drumm, P. V. Duppen, A. Evensen, G. Focker, A. Jokinen, O. Jonsson, E. Kugler, H. Ravn, Pulse shape of the isolde radioactive ion beams, Nuclear Instruments and Methods in Physics Research Section B: Beam Interactions

1. with Materials and Atoms 126 (1-4) (1997) 130-134. doi:10. 1016/s0168-583x(96)01025-7

[28] M. Fadil, CRIBE JRA Eurisol, First Periodic Scientific Report, https://eurisol-jra.in2p3.fr/?page_id=79 [Online; accessed 15-December-2018] (2018). 